\title{
Massaging the message: an abundance of communication pathways used and abused
}

\author{
David F. Albertini ${ }^{1}$
}

Published online: 22 February 2021

(C) The Author(s), under exclusive licence to Springer Science+Business Media, LLC part of Springer Nature 2021

From the formative stages of training in reproductive physiology, no matter what career direction was ultimately pursued, an appreciation for the importance of communication between cells, tissues, and organ systems was a given. The stuff of classical endocrinology best exemplifies communication over "long" distances. And 50 years ago first principles were guided by the one hormone-one receptor notion buttressed by the sequelae of second messenger production and action at what was then regarded as a mostly nuclear (genomic) affair. Messaging in the context of reproductive physiology broadly and human ARTs in particular is a far more complicated affair today!

This transformation in principle and practice has been gradually brought to light by advances in cell and molecular biology, the primary outcome of which is an understanding of signaling between cells at ever diminishing distances. First came the "reach out and touch someone strategy" where physical approximation of cells to one another brought with it the opportunity to engage in a dialog between like or unlike partners in a conversation involving secreted factors and their cognate receptors. Enter the wonderful world of paracrinology. More than adding another layer of complexity to the endocrine motif, getting and receiving messages from cells proximal or distal to where the action takes place now invokes a myriad of intracellular responses responsible for eliciting changes in target cell behavior that collectively conspire to maintain homeostasis.

Reproductive medicine and biology have, and will continue, to rely upon deepening our understanding of how communication pathways of various kinds together dictate an organism's ability to reproduce. If mismanaged, subfertility or

David F. Albertini

eicjarg@gmail.com

1 Bedford Research Foundation, Bedford, MA, USA sterility result. Massaging the message in even a slightly errant way has dire consequences.

Fertilization itself is one of the best examples of how communication gone awry halts the process of reproduction up front and personal. The forces that drive motility constitute an example of intracellular communication between the dynein motor molecules that bridge and translocate the axonemal microtubules. Recently, and at a most reductionist level of approach, molecular details surrounding the protein-protein interactions responsible for normal and abnormal wave forms have been uncovered [1]. Insights provided from studies of this kind in animal models provide direction to ongoing genetic and molecular investigations seeking potentially treatable conditions of male infertility already recognized to be based in the axoneme motility mechanism [2].

But Mother Nature's introduction of a cast of characters capable of initiating and moderating conversations within the reproductive tract shows no signs of slowing down. On the male side, it has become apparent that as sperm transit from epididymis and out into the real world, baggage is accrued via the delivery of important modifying molecules utilizing extracellular vesicles (EVs), or exosomes [3]. That these message-bearing vesicles are operating at many levels of the reproductive axis in males and females has gained increasing attention. One well-studied example emphasizes their importance during the process of implantation [4].

With this all said, Machtinger and her colleagues bring our readership up to speed with their thoughtful and thorough review this month (Extracellular vesicles and female reproduction https://doi.org/10.1007/s10815-020-02048). Deploying EVs in the many circumstances where cells in the reproductive tract must also entertain, embrace, decipher, and process signals received through other modalities - the endocrine, the paracrine, the autocrine gang of 3-makes for a challenging scenario where ambiguities in the final output might not come as a surprise. Just how all this information gets translated into appropriate behaviors has come under the purview of "connectomics." 
Fast forward to today's world of communication strategies amidst pandemics and the ongoing practice of human ARTs, and some likenesses emerge with a stretch of the imagination (The virtual infertility community: a qualitative analysis of patient experiences shared on Instagram, https://doi.org/10. 1007/s10815-020-02028). Enabled in large part by transitioning from printed word of old to the world of social media, discerning useful and reliable information from the spectrum of misinformation or frank deception is the task at hand. And one area within the realm of human ARTs that continues to command attention is that of oocyte cryopreservation (OCP), whether for solid medical indications as we have seen in cases of threatened long term fertility, or in the arena of social egg freezing. Take for example the recent report by Pacia and Howard from The Hastings Center in New York querying the sudden uptick in OCP during the SARS CoV-2 pandemic (http://www. bioethics.net/2021/01/surprising-surge-of-egg-freezingduring-the-pandemic-raises-ethical-question).

Granted the ongoing pandemic has added a tier of concern among would-be-parents regarding their current and future health matters. But taking a close look at this and other ART offerings immediately draws attention to the marketplace and how it has changed since the onset of the pandemic. Advertising as an online medium for communication is more widespread than ever, and with time on their hands, consumers now find themselves deeply embedded in the fact or fallacy, or simply exaggeration decision making process not unlike that experienced by a patient undergoing COSmaybe.
Bayefsky's call for a closer examination of the power of advertising is a message that needs some massaging, but in a way that will make the future of human ARTs a useful and trustworthy course of medical treatment for all who seek it [5].

We close in welcoming Dr. Mark Trolice to the editorial board this month. As always we look forward to your continued support of JARG and encourage any and all feedback from our readership.

\section{References}

1. Gadadhar S, Alvarez Viar G, Hansen JN, Gong A, Kostarev A, IalyRadio C, et al. Tubulin glycylation controls axonemal dynein activity, flagellar beat, and male fertility. Science. 2021;371:6525.

2. Linck RW, Chemes H, Albertini DF. The axoneme: the propulsive engine of spermatozoa and cilia and associated ciliopathies leading to infertility. J Assist Reprod Genet. 2016;33(2):141-56.

3. James ER, Carrell DT, Aston KI, Jenkins TG, Yeste M, Salas-Huetos A. The role of the epididymis and the contribution of epididymosomes to mammalian reproduction. Int $\mathrm{J}$ Mol Sci. 2020;21(15). https://doi.org/10.3390/ijms21155377.

4. Evans J, Rai A, Nguyen HPT, Poh QH, Elglass K, Simpson RJ, et al. Human endometrial extracellular vesicles functionally prepare human Trophectoderm model for implantation: understanding bidirectional maternal-embryo communication. Proteomics. 2019;19(23): e1800423.

5. Bayefsky MJ. Legal and ethical analysis of advertising for elective egg freezing. J Law Med Ethics. 2020;48(4):748-64.

Publisher's note Springer Nature remains neutral with regard to jurisdictional claims in published maps and institutional affiliations. 